\title{
Comparing Effectiveness of Motivational Signage for Promoting Physical Activity between Two Difference Story buildings
}

Junyoung Hong ${ }^{1}$, Aram Yoon ${ }^{2}$, Soon-Mi Choi ${ }^{3}$, Dongwoo Hahn ${ }^{3}$, Minsoo Kang 4 , and Sukho Lee ${ }^{1}$

${ }^{1}$ Laboratory of Human Performance; Department of Curriculum \& Kinesiology; Texas A\&M University- San Antonio, San Antonio, TX. ${ }^{2}$ Laboratory of Health \& Wellness; Department of Curriculum \& Pedagogy; Texas A\&M International University, Laredo, TX. ${ }^{3}$ University of Texas at Austin, Austin, TX. ${ }^{4}$ Middle Tennessee State University, Murfreesboro, TN.

Category: Masters

Advisor / Mentor: Lee, Sukho (slee@tamusa.tamus.edu)

\section{ABSTRACT}

Regular physical activity can reduces the risk of leading cause of diseases including obesity, high blood pressure, cardiovascular, and even some types of cancer. Taking stairs can be a low-cost and relatively easy way to increase daily physical activity. Posting motivational signage containing health promotional information nearby elevator and wall of stairs can be an effective intervention to encourage people to use more stairs resulting in increasing daily physical activities. The purpose of this study was to compare the effectiveness of motivational signage placed in two different story buildings (three vs. Four-story buildings) at the University for encouraging stair use. This study was divided into 3-phases; 1) The first phase (1st week) provides baseline information, 2) the second phase (2nd week) was the intervention with the motivational, and 3) the third phase (3rd week) was the follow up after all motivational signage were removed. The observers were inconspicuously sitting near at the each designated sites without interacting with the participants and recorded usage of either stair or elevator. Each week, observations were taken on the same weekdays (from Monday to Friday) and during the same time of day (7:30 am to 4:30 pm), at both sites. The adjusted odd ratios (OR) and 95\% confidence intervals (CI) for stair and elevator use were calculated using logistic regression procedures. A total of 18,927 people were participated in this study. In phase $1,47.07 \%$ of our participants used the stairs. Total percentage of stair usage was significantly increased during the intervention phases and well maintained in phase $3(54.73 \%, \mathrm{OR}=1.35 ; 95 \% \mathrm{CI}=1.25$ -1.45 in phase 2 and $53.51 \%, \mathrm{OR}=.93 ; 95 \% \mathrm{CI}=.87-.99$ in phase 3). Stair usage in three-story building was significantly higher than four-story building ( $\mathrm{OR}=3.46 ; 95 \% \mathrm{CI}=3.21-3.72)$. The motivational signage can be used as an effective method for increasing daily physical activity on the university campus. Buildings with different stories need to be considered in promoting stair use.

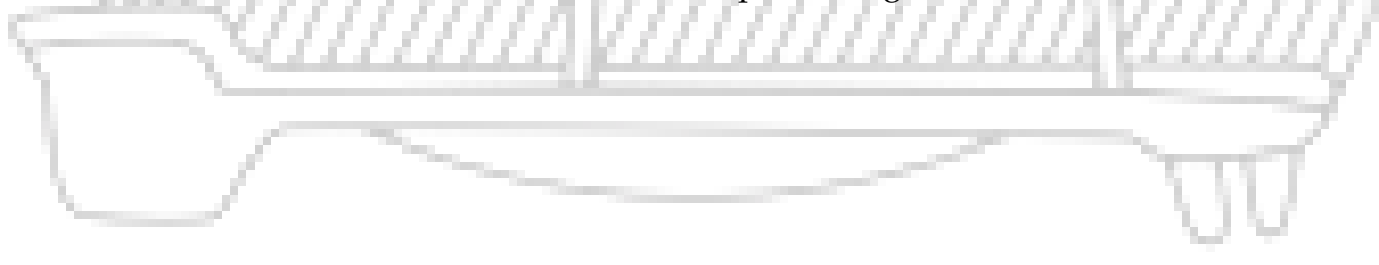

